\title{
Spontaneous recanalization of a completely occluded saphenous vein graft following unsuccessful percutaneous coronary intervention is
}

\author{
Erkan Yildirim a , Baris Bugan ${ }^{\mathrm{b}, *}$, Uygar Cagdas Yuksel ${ }^{\mathrm{a}}$, Suat Gormel ${ }^{\mathrm{a}}$, Yalcin Gokoglan ${ }^{\mathrm{a}}$, Murat Celik ${ }^{\mathrm{a}}$ \\ a Gulhane Military Medical Academy, Department of Cardiology, Ankara, Turkey \\ b Girne Military Hospital, Cardiology Service, Girne, Turkish Republic of Northern Cyprus, Cyprus
}

\section{A R T I C L E I N F O}

\section{Article history:}

Received 24 June 2016

Received in revised form 19 December 2016

Accepted 23 December 2016

Available online 28 December 2016

\section{Keywords:}

Angioplasty

Myocardial infarction

Thrombolysis

Saphenous vein graft

\begin{abstract}
A B S T R A C T
Saphenous vein graft (SVG) infarctions usually occur in degenerated vein grafts filled with atherothrombotic debris. The success rate of percutaneous coronary intervention (PCI) to SVG decreases because of their greater content of thrombus and other material such as necrotic debris. In this case report, we present an unusual case of a patient presenting with total occlusion of an Aorta-Posterior descending (Ao-Pd) SVG during inferior acute myocardial infarction, which spontaneously recanalized over fifteen days period following failed PCI.

(C) 2017 The Society of Cardiovascular Academy. Production and hosting by Elsevier B.V. All rights reserved. This is an open access article under the CC BY-NC-ND license (http://creativecommons.org/licenses/by-nc-nd/4.0/).
\end{abstract}

\section{Introduction}

Acute myocardial infarction resulting from saphenous vein graft (SVG) occlusion occurs not infrequently in patients who have undergone coronary artery bypass graft surgery $(\mathrm{CABG}){ }^{1}$ Studies have suggested that SVG occlusion occurs at a rate of approximately $10 \%$ in the first month and up to $20 \%$ in the first year, with a $2 \%$ annual risk for the first five years. ${ }^{2}$ Recurrent infarction may occur in as many as $29 \%$ of patients in 10 years following CABG. ${ }^{3}$ SVG infarctions usually happen in degenerated vein grafts filled with atherothrombotic debris. The success rate of PCI to SVG decreases because of their greater content of thrombus and other material such as necrotic debris. ${ }^{4,5}$ In this case report, we present an unusual case of a patient presenting with total occlusion of an Aorta-Posterior descending (Ao-Pd) SVG during inferior acute myocardial infarction, which spontaneously recanalized over fifteen days period following failed $\mathrm{PCI}$.

\section{Case report}

A 76-year old man with a history of known atherosclerotic heart disease (CABG 5 years ago), hypertension and hyperlipidemia admitted to our center with atypical chest pain. Fifteen days before admission, the patient was hospitalized for acute inferior myocardial infarction (Fig.

\footnotetext{
it Peer review under responsibility of The Society of Cardiovascular Academy.

* Corresponding author at: Corlu Goverment Hospital, Cardiology Service, 59850, Corlu, Tekirdag, Turkey.

E-mail address: bbugan@hotmail.com (B. Bugan).

Peer review under responsibility of The Society of Cardiovascular Academy.
}

1A) and had a coronary angiography in another center. He had retrosternal chest pain that radiates to left arm; associated with profuse sweating and vomiting. In his physical examination mild systolic ejection murmur was noticed at the left sternal border. Moist rales was heard on $1 / 3$ lower pulmonary areas. The electrocardiography revealed normal sinus rhythm with pathologic Q waves, 2-3 mm ST segment elevation and T wave inversions in leads DII, III and aVF. Cardiac markers such as Troponin I, creatine kinase MB isoform and lactate dehydrogenase enzyme elevated in the patient's serum. Prompt coronary angiography was performed due to ongoing chest pain. This angiogram showed complete thrombotic occlusion of the SVG to the posterior descending artery (PDA) (Fig. 1B), and 70\% stenosis in obtuse marginal (OM) branch of circumflex artery (CFX). Native left anterior descending artery (LAD) and native right coronary artery (RCA) were occluded in mid-portion. Left internal mammary artery (LIMA) graft to LAD was patent. The operators had decided to perform primer percutaneous coronary intervention (P-PCI) to Ao-Pd SVG, but the intervention was resulted with failure after several attempts (Fig. 2A). They hospitalized the patient for 3 days. During the hospitalization he received $300 \mathrm{mg}$ oral aspirin and 7000 unit intravenous unfractionated heparin the first day before the procedure and oral dual antiplatelet therapy (aspirin $100 \mathrm{mg} /$ day and clopidogrel $75 \mathrm{mg} /$ day) and anticoagulation therapy (subcutaneous enoxaparin $6000 \mathrm{IU}$ once a day) after the failed intervention. The patient was discharged on optimal anti-ischemic therapy with aspirin $100 \mathrm{mg}$ and clopidogrel $75 \mathrm{mg}$ per day as the only antiplatelet agents without any anticoagulant therapy.

Fifteen days after the initial hospitalization, the patient developed chest pain again and admitted to our emergency department. Physical examination revealed an apical grade $2 / 6$ systolic murmur on 


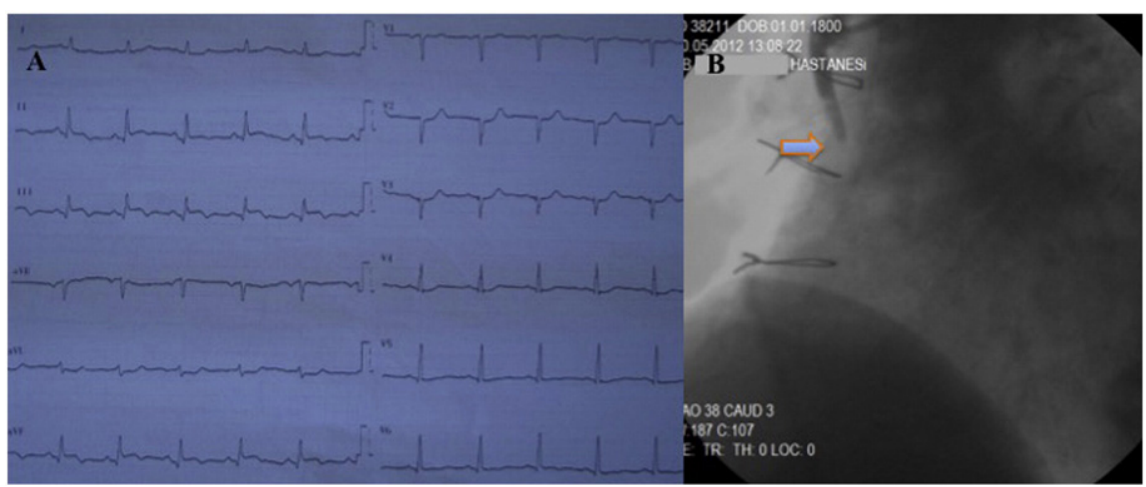


artery (B). ECG, Electrocardiogram; SVG, saphenous vein graft.

auscultation. Jugular venous distention and pretibial edema were not remarkable, and auscultation revealed clear lungs. His laboratory findings revealed insignificantly elevated cardiac biomarkers [troponin I (TnI) and creatine kinase-MB isoenzyme (CK-MB)] with mild ST depression in the inferior leads on his electrocardiography. Transthoracic echocardiography revealed a mild hypokinesia of midapical inferiorposterior wall with mild LV dilatation (LV ejection fraction, 50\%). We decided to perform fractional flow reserve (FFR) to $70 \%$ stenosis in $\mathrm{OM}$ branch of CFX. Coronary angiogram surprisingly showed complete patency of the previously occluded SVG to the PDA (Fig. 2B) with a complete angiographic resolution of the thrombus. The stenosis in CFX assessed noncritical with FFR, and the patient discharged on medical therapy. He was followed up at the outpatient clinic for two years uneventfully.

\section{Discussion}

Graft failure may occur due to thrombosis, fibro-intimal hyperplasia, and atherosclerosis, respectively. There is general agreement that vein graft atherosclerosis differs from arterial lesions regarding temporal and histological changes. Vein graft atherosclerosis is more rapid, with diffuse concentric changes and a less noticeable fibrous cap, making venous plaques more vulnerable to rupture and subsequent thrombus formation. ${ }^{6}$ The success rate of PCI to SVG decreases because of their greater content of thrombus and other material such as necrotic debris. ${ }^{4,5}$ Their increased content of thrombus and another material such as necrotic debris predisposes them to a greater propensity for distal embolization and no-reflow phenomenon after intervention. ${ }^{7}$ In SVG infarct cases, the decision to perform an intervention is made after taking into consideration infarct size and duration, patient condition, thrombus burden, and vessel anatomy. Optimal strategy sometimes can be controversial in SVG occlusions. ${ }^{4}$ These patients usually have three options: i) PCI of the severely diseased native vessels, ii) repeat CABG or iii) medical therapy alone.

This report demonstrates the rare phenomenon of complete angiographic thrombus resolution and reperfusion of a thrombotically occluded SVG following treatment with aspirin and clopidogrel as the only outpatient antiplatelet therapy. It is an example of physiological capability for spontaneous fibrinolysis of even very large thrombi in completely occluded SVGs. Whereas catheter-based interventions are the treatment of choice, conservative strategy powered by new sparkling dual antiplatelet and anticoagulant agents await to be just an alternative option after failed revascularization.

According to the studies in pigs, the local profibrinolytic response in coronary arteries, by the net coronary release of tissue-type plasminogen activator (t-PA) following induced myocardial ischemia seems to be a useful defense against coronary thromboembolic events. Higher levels of t-PA are noted in ST-segment elevation myocardial infarction and are associated with worse prognosis. ${ }^{8}$ Possible mechanisms of spontaneous recanalization include the release of factors, activators, and inhibitors of fibrinolysis, from the endothelium, neutrophils and monocytes. ${ }^{9}$

After acute coronary syndrome, dual antiplatelet therapy (DAPT) is the standard of care for both invasive management with percutaneous intervention and noninvasive (medical) management. Plaque stability, prevention of graft closure, and secondary thrombosis form the basis for using a second antiplatelet drug, whereas the additional risk of bleeding and lack of conclusive evidence should also be considered. ${ }^{10}$

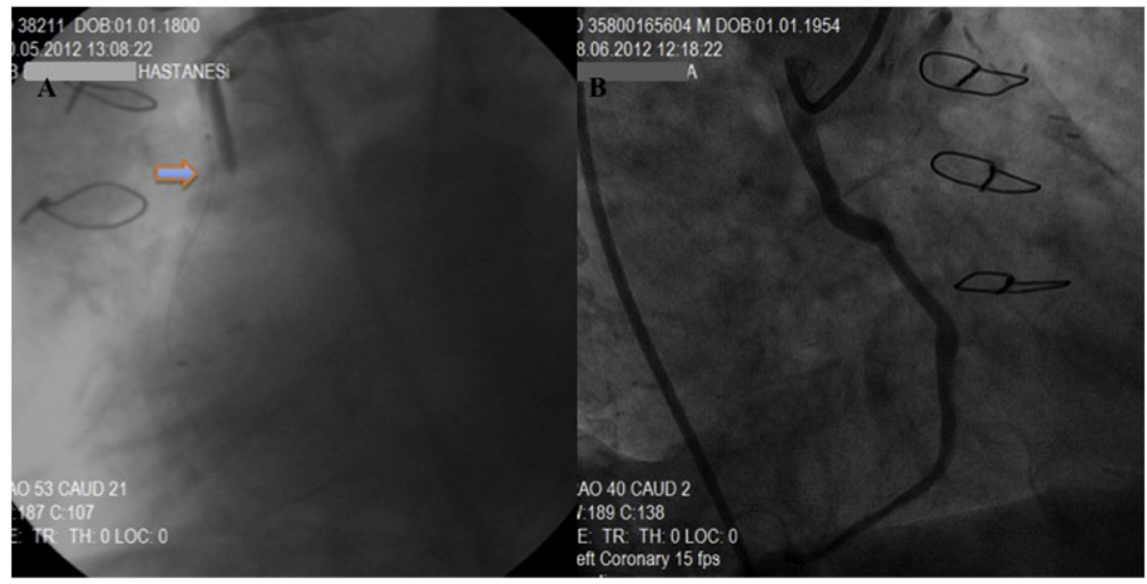

Fig. 2. Failed P-PCI to SVG (A). Complete patency of the previously occluded SVG (B). P-PCI, primer percutaneous coronary intervention; SVG, saphenous vein graft. 
Large, multicenter, randomized clinical trials are needed to investigate definitively the role of DAPT in patients with occluded saphenous vein graft following unsuccessful PCI during acute myocardial infarction.

Glycoprotein (GP) IIb/IIIa antagonists are potent inhibitors and they are advised to be considered for bailout therapy if there is angiographic evidence of massive thrombus, slow or no-reflow or a thrombotic complication with class IIa recommendation according to ESC guidelines. ${ }^{11}$ In our case after demonstrating thrombotic lesion in the saphenous graft ignoring Gp IIb/IIla antagonists beside DAPT and unfractioned heparin may be the reason for the failed intervention.

Coronary spasm or dynamic obstruction is an infrequent but important cause of coronary syndromes in native arteries however in venous grafts it is an unusual situation and its clinical relevance remains to be established. ${ }^{12}$ The cause of spasm in venous grafts is smooth muscle cell hyperreactivity just like coronary spasm and it is associated with a high prevalence of migraine and Raynaud's phenomenon. ${ }^{13}$ In our case dynamic obstruction may be the reason for total occlusion in saphenous vein although it is a less probable physiological mechanism.

According to a study by Cheol Whan Lee et al. for determinants and prognostic significance of spontaneous coronary recanalization in acute myocardial infarction in 196 consecutive patients; faster coronary flow, smaller infarct size, and a better clinical outcome after primary angioplasty are associated with spontaneous recanalization. ${ }^{14}$ Preserved ejection fraction and small infarct size are concordant findings in our patient with Cheol Whan Lee's study in the way of good prognosis relieving coronary syndrome.

\section{Conclusion}

We present a unique case of spontaneous reperfusion of SVG, treated medically and discharged on only dual anti-platelet agents. Although modern medical era seems prone to percutaneous approach for acute total occlusions, we see that while deciding the management strategy in thrombus rich SVG lesions during acute coronary syndromes more conservative approaches can be the optimal choice. As mentioned before in an English proverb "nature, time and patience are three great physicians."

\section{Conflict of interest}

There is no conflict of interest.

\section{References}

1. Pereg D, Fefer P, Samuel M, et al. Native coronary artery patency after coronary artery bypass surgery. JACC Cardiovasc Interv 2014;7:761-767.

2. Fitzgibbon GM, Kafka HP, Leach AJ, Keon WJ, Hooper GD, Burton JR. Coronary artery bypass grafting fate and angiographic follow-up of 5065 grafts related to survival and reoperation in 1388 patients in 25 years. J Am Coll Cardiol 1996;28:616-626.

3. Brooks MM, Alderman EL, Bates E, et al. The final 10-year follow-up results from the BARI randomized trial. J Am Coll Cardiol 2007:49:1600-1606.

4. Shavadia J, Norris CM, Graham MM, Verma S, Ali I, Bainey KR. Symptomatic graft failure and impact on clinical outcome after coronary artery bypass grafting surgery: results from the Alberta provincial project for outcome assessment in coronary heart disease registry. Am Heart J 2015;169:833-840.

5. Hess CN, Lopes RD, Gibson CM, et al. Saphenous vein graft failure after coronary artery bypass surgery: insights from PREVENT IV. Circulation 2014;21:1445-1451.

6. Hassantash SA, Bikdeli B, Kalantarian S, Sadeghian M, Afshar H. Pathophysiology of aortocoronary saphenous vein bypass graft disease. Asian Cardiovasc Thorac Ann 2008;16:331-336.

7. Maia F, Costa Jr JR, Abizaid A, et al. Preliminary results of the INSPIRE trial with the novel MGuard stent system containing a protection net to prevent distal embolization. Catheter Cardiovasc Interv 2010;76:86-92.

8. Jansson JH, Olofsson BO, Nilsson TK. Predictive value of tissue plasminogen activator mass concentration on long-term mortality in patients with coronary artery disease: a 7-year follow-up. Circulation 1993;88:2030-2034.

9. Osterlund B, Andersson B, Haggmark S, et al. Myocardial ischemia induces coronary t-PA release in the pig. Acta Anaesthesiol Scand 2002;46:271-278.

10. Montalescot G, Sabatine MS. Oral dual antiplatelet therapy: what have we learnt from recent trials? Eur Heart J 2016;21(37):344-352.

11. Task Force on the management of ST-segment elevation acute myocardial infarction of the European Society of Cardiology (ESC), Steg PG, James SK, et al. ESC guidelines for the management of acute myocardial infarction in patients presenting with ST-segment elevation. Eur Heart J 2012;33:2569-2619.

12. Porto I, Burzotta F, Mongiardo R, Crea F. Left main and saphenous vein graft spasm: an unusual association. Int J Cardiol 2005;99:133-134.

13. Maseri A, Beltrame JF, Shimokowa H. Role of coronary vasoconstriction in ischemic heart disease and search for novel therapeutic targets. Circ J 2009;73:394-403.

14. Lee CW, Hong MK, Lee JH, Yang HS, Kim JJ, Park SW, Park SJ. Determinants and prognostic significance of spontaneous coronary recanalization in acute myocardial infarction. Am J Cardiol 2001;87:951-954. 